
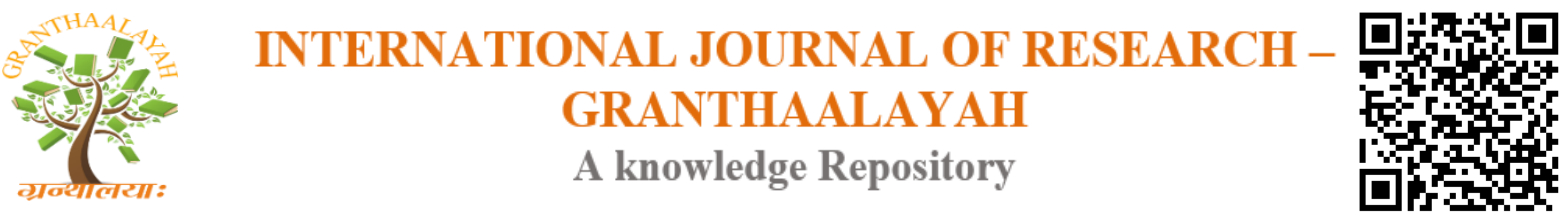

Science

\title{
EXPERIMENTAL STUDY AND ANALYSIS OF SOLAR AIR HEATER USING OF VARIOUS INLET TEMPERATURES
}

\author{
Deeksha Vishwakarma ${ }^{1}$, Prof. Jyoti Kale ${ }^{2}$ \\ ${ }^{1}$ M. Tech. (Student), Heat Power and Thermal, Department of Mechanical Engineering, VITS, \\ Jabalpur (M.P.), India \\ ${ }^{2}$ Assistant Professor, Department of Mechanical Engineering, VITS, Jabalpur (M.P.), India
}

\begin{abstract}
In this paper, we are make the experimental setup and assembled the various components such as, flats plate glass, wood, collector, inlet port and outlet port. The collector are generated the hot air with the help solar radiation. The collector are aching the air from inlet port and finally hot air are out through outlet port. We are measured the maximum outlet hot air Temperature in ${ }^{\circ} \mathrm{C}$.

Keywords: Flats Plate Glass; Wood; Collector.

Cite This Article: Deeksha Vishwakarma, and Prof. Jyoti Kale. (2017). "EXPERIMENTAL STUDY AND ANALYSIS OF SOLAR AIR HEATER USING OF VARIOUS INLET TEMPERATURES." International Journal of Research - Granthaalayah, 5(10), 76-80. https://doi.org/10.29121/granthaalayah.v5.i10.2017.2271.
\end{abstract}

\section{Introduction}

By solar air heater, we are obtaining the hot air. The upper portion of solar air heater are covered with the help of glass, inlet air are heated by solar radiation which are interring with the help of glass.

\section{Experimental Setup}

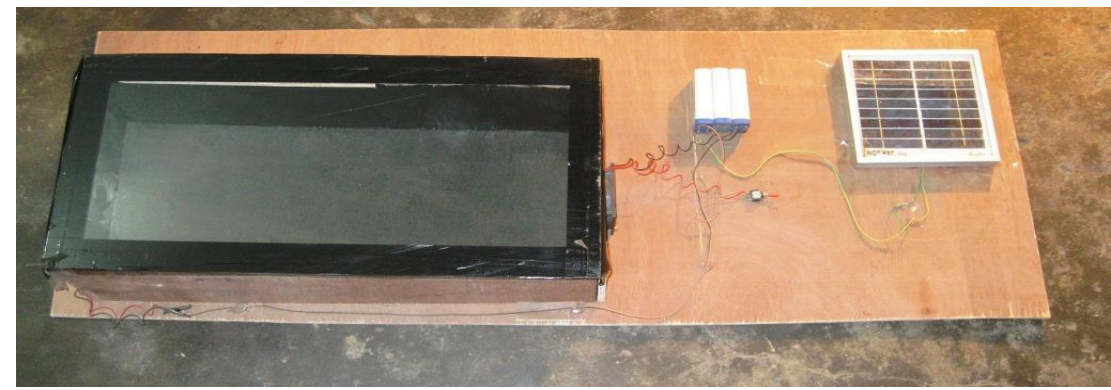

Figure 1: Experimental setup 


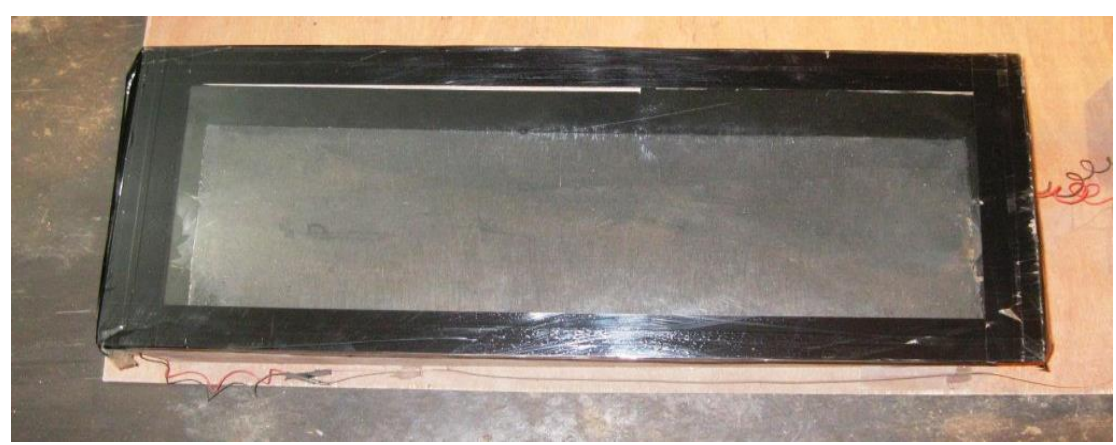

Figure 2: solar collector

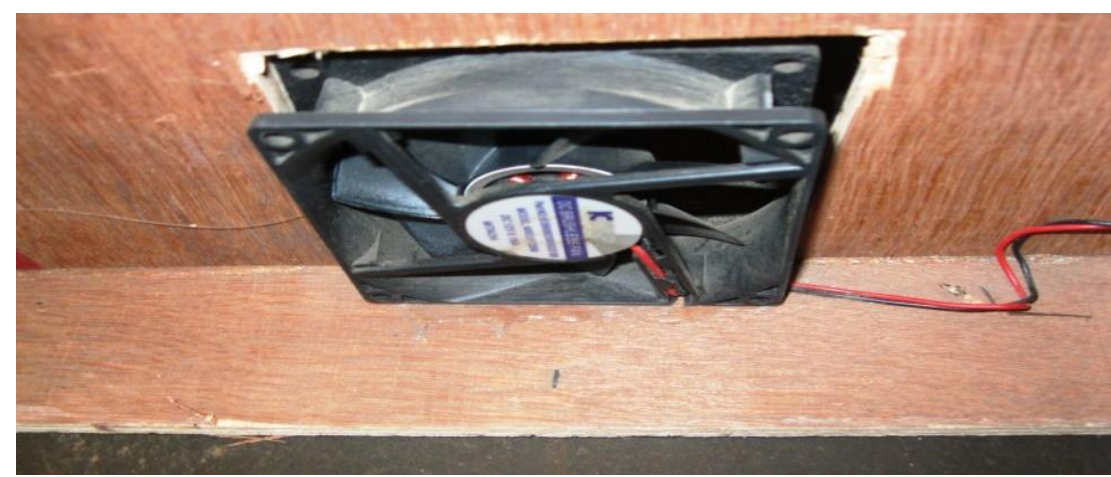

Figure 3: D.C. fan

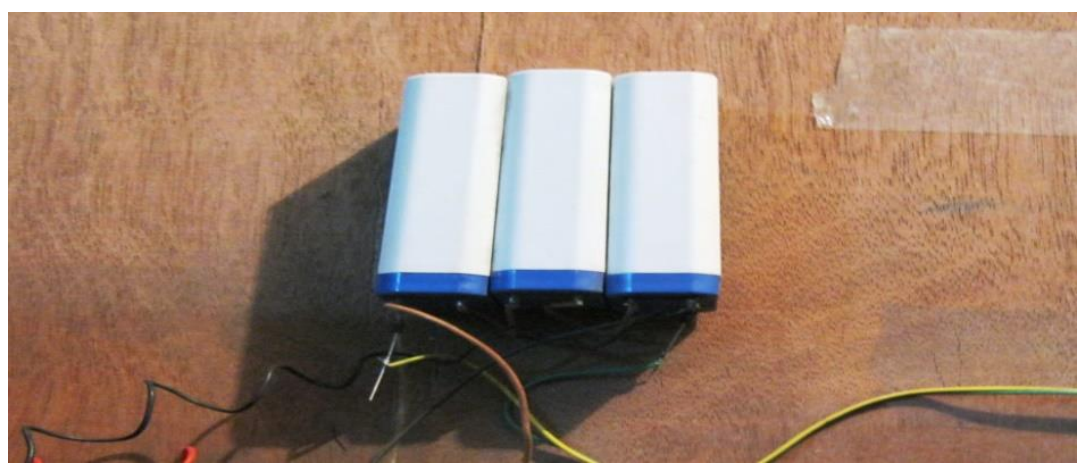

Figure 4: battery

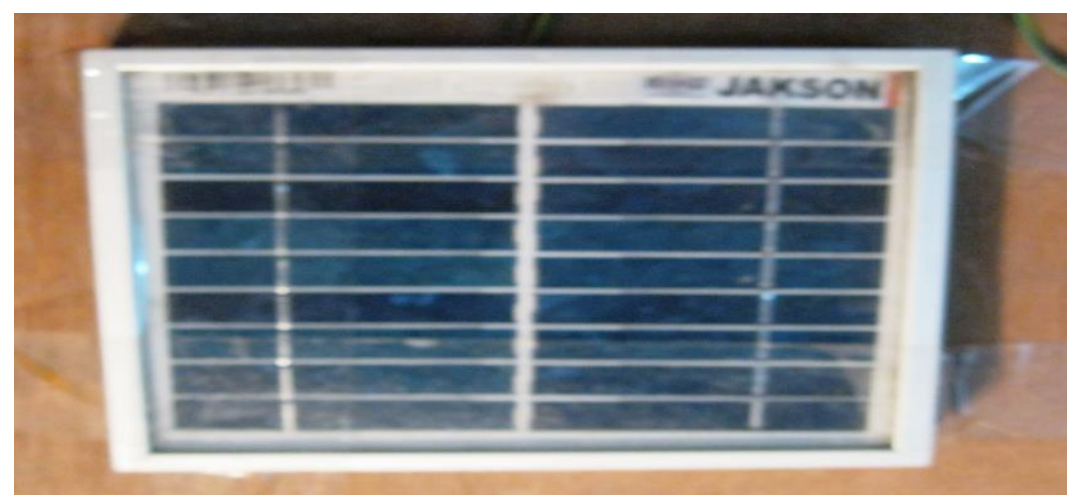

Figure 5: photovoltaic cells 


\section{Results and Discussion}

Table 1: Mass air flow rate at $0.30 \mathrm{~kg} / \mathrm{sec}$ using of simple absorber trays forced convection

\begin{tabular}{|l|l|l|l|}
\hline Sr.No. & Time In hours & Temperature in ${ }^{\circ} \mathbf{C}$ at inlet & Temperature in ${ }^{\circ} \mathbf{C}$ at outlet \\
\hline 1 & $10: 00$ & 30 & 41 \\
\hline 2 & $11: 00$ & 31 & 44 \\
\hline 3 & $12: 00$ & 32 & 52 \\
\hline 4 & $13: 00$ & 40 & 55 \\
\hline 5 & $14: 00$ & 41 & 48 \\
\hline 6 & $15: 00$ & 36 & 49 \\
\hline 7 & $16: 00$ & 32 & 45 \\
\hline
\end{tabular}

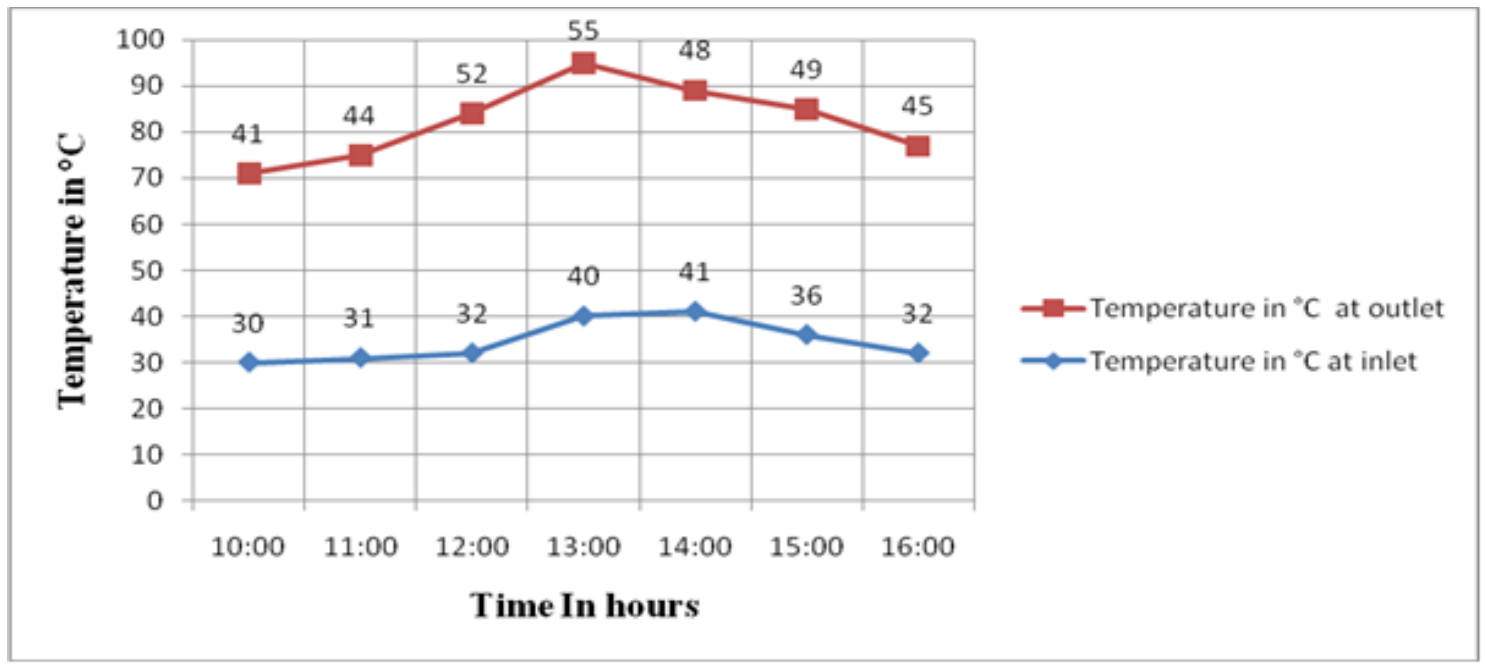

Figure 6: Mass air flow rate at $0.30 \mathrm{~kg} / \mathrm{sec}$ using of simple absorber trays forced convection

Table 2: Mass air flow rate at $0.40 \mathrm{~kg} / \mathrm{sec}$ using of simple absorber trays forced convection

\begin{tabular}{|l|l|l|l|}
\hline Sr.No. & Time In hours & Temperature in ${ }^{\circ} \mathbf{C}$ at inlet & Temperature in ${ }^{\circ} \mathbf{C}$ at outlet \\
\hline 1 & $10: 00$ & 29 & 44 \\
\hline 2 & $11: 00$ & 30 & 47 \\
\hline 3 & $12: 00$ & 31 & 55 \\
\hline 4 & $13: 00$ & 42 & 57 \\
\hline 5 & $14: 00$ & 41 & 49 \\
\hline 6 & $15: 00$ & 37 & 48 \\
\hline 7 & $16: 00$ & 32 & 44 \\
\hline
\end{tabular}




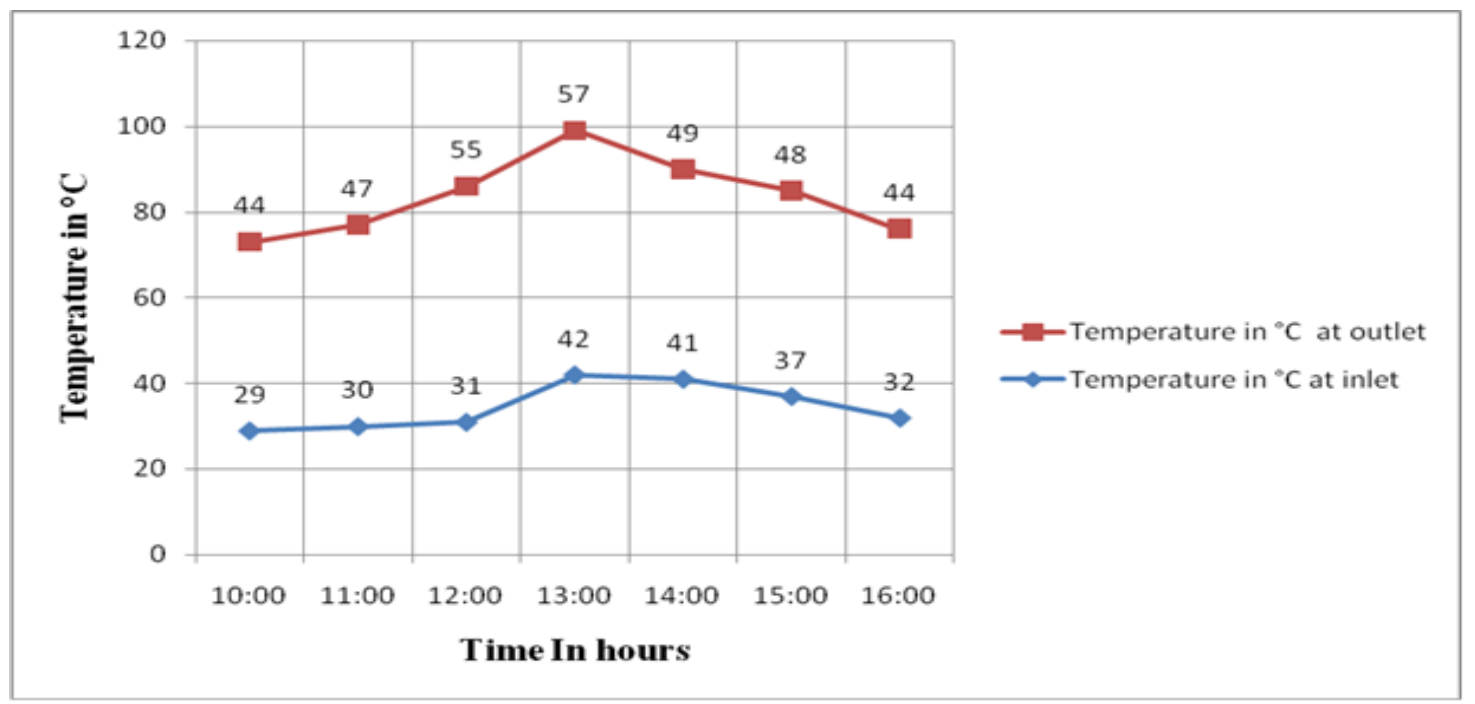

Figure 7: Mass air flow rate at $0.40 \mathrm{~kg} / \mathrm{sec}$ using of simple absorber trays forced convection

Table 3: Mass air flow rate at $0.50 \mathrm{~kg} / \mathrm{sec}$ using of simple absorber trays forced convection

\begin{tabular}{|l|l|l|l|}
\hline Sr.No. & Time In hours & Temperature in ${ }^{\circ} \mathbf{C}$ at inlet & Temperature in ${ }^{\circ} \mathbf{C}$ at outlet \\
\hline 1 & $10: 00$ & 30 & 48 \\
\hline 2 & $11: 00$ & 32 & 50 \\
\hline 3 & $12: 00$ & 33 & 57 \\
\hline 4 & $13: 00$ & 43 & 62 \\
\hline 5 & $14: 00$ & 41 & 56 \\
\hline 6 & $15: 00$ & 36 & 52 \\
\hline 7 & $16: 00$ & 32 & 49 \\
\hline
\end{tabular}

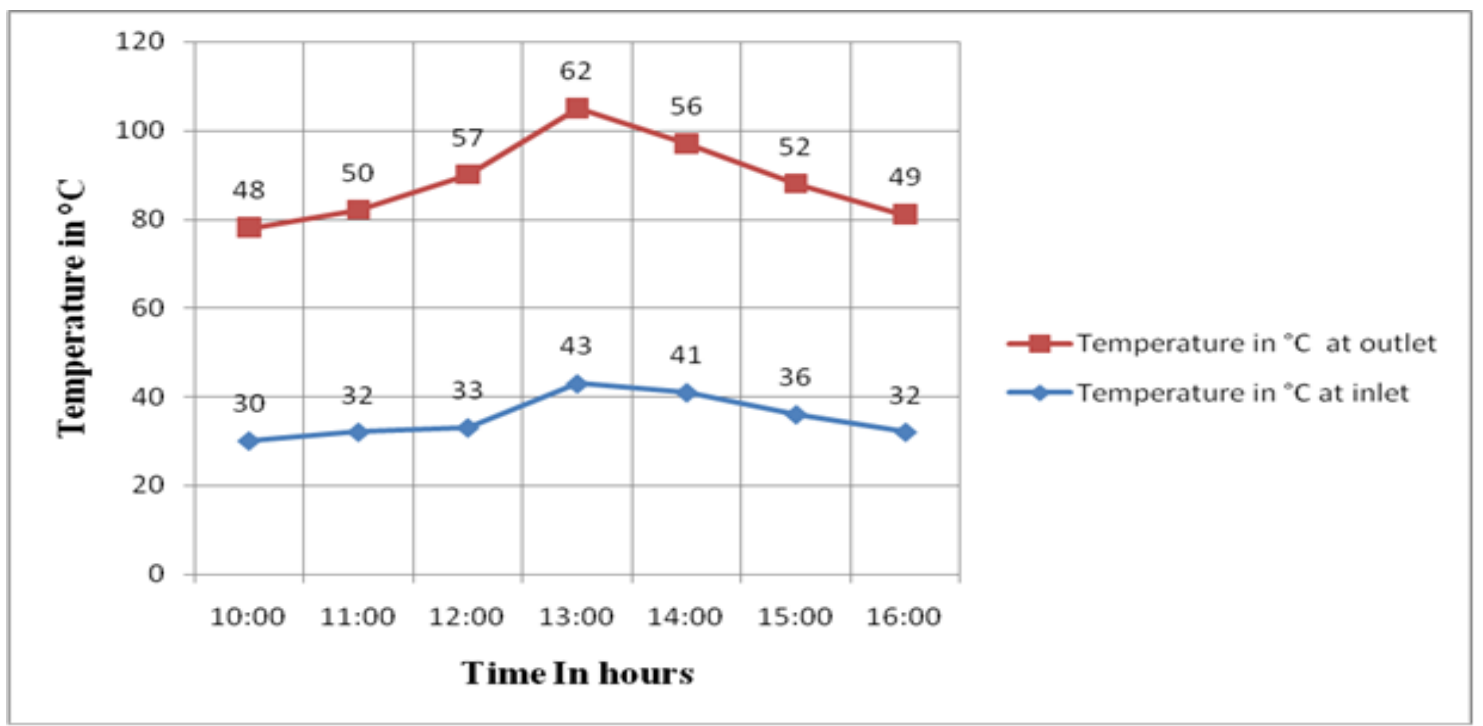

Figure 8: Mass air flow rate at $0.50 \mathrm{~kg} / \mathrm{sec}$ using of simple absorber trays forced convection 


\section{Conclusion}

In this paper, we are measured the various temperatures using of digital temperature measurement devices. We are finding out the maximum $62{ }^{\circ} \mathrm{C}$ Temperature at outlet and times are 13:00, the Mass air flow rate are $0.50 \mathrm{~kg} / \mathrm{sec}$ using of simple absorber trays forced convection, which are shown in table .3 .

\section{References}

[1] A. Hachemi et al. Experimental study of thermal performance of offset rectangular plate fin absorber plates Renewable Energy 17(1999) pp.371-384.

[2] A. Georgiev et al. testing solar collectors as an energy source for a heat pump Renewable Energy 33 (2008) pp.832-838.

[3] Bessler WF et al. Solar assisted heat pumps for residential use. ASHRAE J 1980; pp.59-63.

[4] Cemil Yamal et al. Theoretical investigation of a humidification dehumidification desalination system configured by a double-pass flat plate solar air heater Desalination 205 (2007) pp.163-177

[5] Chaturvedy SK et al. Energy conservation potential of large capacity Solar-assisted heat pumps for low temperature IPH applications. Trans ASME, J Sol Energy Eng 1985 pp.286-296. 